\title{
From "fat" graphs to metric graphs: the problem of boundary conditions
}

\author{
G. F. Dell'Antonio' ${ }^{1}$, A. Michelangeli ${ }^{2}$ \\ ${ }^{1}$ Sapienza, Rome, Italy and \\ SISSA, Via Bonomea 265, 34136, Trieste, Italy \\ ${ }^{2}$ SISSA, Via Bonomea 265, 34136, Trieste, Italy and \\ Center for Advanced Studies, Ludwig-Maximilians-Universität München, \\ Geschwister-Scholl-Platz, 1, 80539, Munich, Germany \\ gianfa@sissa.it
}

PACS 03.65.Ge, 02.30.Jr

DOI 10.17586/2220-8054-2015-6-6-751-756

\begin{abstract}
We discuss how the vertex boundary conditions for the dynamics of a quantum particle on a metric graph emerge when the dynamics is regarded as a limit of the dynamics in a tubular region around the graph. We give evidence for the fact that the boundary conditions are determined by the possible presence of a zero-energy resonance. Therefore, the boundary conditions depend on the shape of the fat graph near the vertex. We also give evidence, by studying the case of the half-line, for the fact that on the contrary, in general, adding on a graph a shrinking support potentials at the vertex either does not alter the boundary condition or does not produce a self-adjoint dynamics. Convergence, throughout, is meant in the sense of strongly resolvent convergence.
\end{abstract}

Keywords: quantum billiards, Schrödinger equation.

Received: 1 November 2015

\section{Introduction}

We consider in $\mathbb{R}^{3}$ a star graph $\Gamma$ with vertex at the origin and $N$ "rays" (half-lines) $K^{(n)}, n=1 \ldots N$. We consider also a suitable vicinity of $\Gamma$ ("fat graph"), denoted by $\Gamma_{\varepsilon}$, whose "width" is proportional to $\varepsilon>0$. More concretely, we consider $\Gamma_{\varepsilon}$ as consisting of a junction region contained in a ball $B_{\varepsilon}$ of radius $C \cdot \varepsilon(C>1)$, attached to which there are $N$ tubes" $K_{\varepsilon}^{(n)}, n=1, \ldots, N$, namely $N$ non-intersecting infinite half-cylinders with transversal radius $\varepsilon$, whose axes are the rays $K^{(n)}$.

The limit $\varepsilon \rightarrow 0$ that we have in mind is a homotetic shrinking of $\Gamma_{\varepsilon}$ to its skeleton $\Gamma$.

The internal region may be arbitrary and need not be connected; it may also be fragmented. In the case of graphene, the image in an electronic microscope shows that the density of conducting electrons is essentially localized in a spherical corona of width approximately equal to the diameter of the cylinders.

This may be considered as a result of the combined action of the attraction to the nucleus and of the presence near the nucleus of the valence electrons.

Let $\Delta_{\Gamma_{\varepsilon}}$ be the Laplacian on $\Gamma_{\varepsilon}$ with Dirichlet boundary conditions at $\partial \Gamma_{\varepsilon}$.

We denote by $\lambda_{\varepsilon}>0$ and $\xi_{\varepsilon}^{(1)}$, respectively, the lowest eigenvalue and the corresponding normalized eigenfunction of the two-dimensional negative Laplacian on a disk with Dirichlet boundary conditions. By scaling $\lambda_{\varepsilon} \sim \varepsilon^{-2} \lambda$.

We define the following:

$$
H_{\varepsilon}:=-\Delta_{\Gamma_{\varepsilon}}-\lambda_{\varepsilon} \mathbf{1}
$$


We have thus obtained a self-adjoint operator on the fat graph $\Gamma_{\varepsilon}$. Dependng on the shape of $\Omega_{\varepsilon}$ for all values of $\varepsilon>0, H_{\varepsilon}$ may possibly have a negative point spectrum and an absolutely continuous spectrum coinciding with $\mathbb{R}^{+}$.

It turns out that a deep understanding of the structure of the limit $\varepsilon \rightarrow 0$ is achieved by means of the notion of zero energy resonance. For the present purposes, we define a zero energy resonance of $H_{\varepsilon}$ as a singularity of the spectral measure of $H_{\varepsilon}$ at the bottom of the continuous spectrum, equivalently, as a singularity in $k^{2}$ at $k=0$ of the resolvent $\left(H_{\varepsilon}-k^{2}\right)^{-1}$.

If the boundary $\partial \Gamma_{\varepsilon}$ is smooth, the singularity at the bottom of the continuous spectrum is of the type $\frac{1}{|k|}$. This corresponds, in our case, due to the special form of the domain $\Gamma_{\varepsilon}$, to a generalized (i.e., distributional) solution $\Phi_{\varepsilon}$ to $H_{\varepsilon} \Phi_{\varepsilon}=0$ which is only square-integrable locally.

There is in fact a one-to-one correspondence between the possible singularities at zero of the resolvent of $H_{\varepsilon}$, due to resonances, and the singularities at zero of the resolvents of each self-adjoint Laplacian on the star graph. The former are non squareintegrable functions that on each cylinder behave, axially, as a constant plus linear function $a_{n}+b_{n} z_{n}$ ( $z_{n}$ is the axial coordinate on the $n$-th cylinder); the latter have the very same behavior on the corresponding rays of the star graph.

\section{Setting the problem up}

We want to study the effect of $\Omega_{\varepsilon}$ on the limit $\varepsilon \rightarrow 0$ by means of the associated problem - we shall call it "the internal region problem" - consisting of the negative Laplacian in the internal region $\Omega_{\varepsilon}$ with boundary conditions that are of some assigned type, denoted by $\alpha$, on the bases of the cylinders, and are of the Dirichlet type on the rest of $\partial \Omega_{\varepsilon}$.

With this choice, we denote by $\mu_{+}\left(\Omega_{\varepsilon}\right)$ and $\mu_{-}\left(\Omega_{\varepsilon}\right)$, respectively, the lowest eigenvalue of the internal region problem when $\alpha=$ Dirichlet or $\alpha=$ Neumann, and by $\mu_{\alpha}\left(\Omega_{\varepsilon}\right)$ the lowest eigenvalue with generic boundary condition $\alpha$ (recall that on the rest of $\partial \Omega_{\varepsilon}$ we always take Dirichlet boundary conditions). Clearly:

$$
\mu_{-}\left(\Omega_{\varepsilon}\right) \leqslant \mu_{\alpha}\left(\Omega_{\varepsilon}\right) \leqslant \mu_{+}\left(\Omega_{\varepsilon}\right),
$$

and each $\mu_{\alpha}\left(\Omega_{\varepsilon}\right)$ scales as $\varepsilon^{-2}$. We also note that by min-max, when one increases $\Omega_{\varepsilon}$, both $\mu_{-}\left(\Omega_{\varepsilon}\right)$ and $\mu_{+}\left(\Omega_{\varepsilon}\right)$ decrease.

Suppose that the internal region problem with a given boundary condition $\boldsymbol{\alpha}$ has a lowest-energy solution given by the eigenfunction $\phi_{\varepsilon}(\mathbf{x})$ and the eigenvalue $\mu_{\alpha}\left(\Omega_{\varepsilon}\right)$, where $\mathbf{x}$ is the three-dimensional coordinate in $\Omega_{\varepsilon}$.

Correspondingly, prolonging $\phi_{\varepsilon}$ by continuity of the function and its derivatives, to a function $\Phi_{\varepsilon}$, also defined also on the external cylinders in such a way that, if $\left(x_{n}, y_{n}\right)$ are the transversal coordinates and $z_{n}$ is the axial coordinate in $K_{\varepsilon}^{(n)}$, then:

$$
\Phi_{\varepsilon}\left(x_{1}, y_{1}, z_{1}, \ldots, x_{N}, y_{N}, z_{N}\right)=\prod_{n=1}^{N} \xi_{\varepsilon}^{(1)}\left(x_{n}, y_{n}\right)\left(a_{n}+b_{n} z_{n}\right), \quad z_{n} \geqslant 0
$$

Fundamental observation: a zero energy resonance for $H_{\varepsilon}$ on $\Gamma_{\varepsilon}$ can occur only if for the associated internal region problem there exists a boundary condition $\alpha$ at the bases of the cylinders such that the first eigenvalue $\mu_{\alpha}\left(\Omega_{\varepsilon}\right)$ of the internal region problem (namely the negative Laplacian inside $\Omega_{\varepsilon}$ with boundary condition $\alpha$ at the bases of the cylinders and Dirichlet boundary conditions on the remaining part of $\partial \Omega_{\varepsilon}$ ) coincides with the lowest 
From "fat" graphs to metric graphs...

eigenvalue $\lambda_{\varepsilon}$ of the negative Laplacian on the cylinders transversal section, namely if

$$
\mu_{-}\left(\Omega_{\varepsilon}\right) \leqslant \lambda_{\varepsilon} \leqslant \mu_{+}\left(\Omega_{\varepsilon}\right) .
$$

Decomposition:

$$
L^{2}\left(\Gamma_{\varepsilon}\right) \cong L^{2}\left(\Omega_{\varepsilon}\right) \oplus\left(\oplus_{n=1}^{N} L^{2}\left(K_{\varepsilon}^{(n)}\right)\right)
$$

$\left(\Omega_{\varepsilon}=\right.$ the central region, $K_{\varepsilon}^{(n)}=$ the cylinders). In turn,

$$
\begin{aligned}
L^{2}\left(K_{\varepsilon}^{(n)}\right) & \cong L^{2}\left(K^{(n)}\right) \otimes L^{2}\left(D_{\varepsilon}\right) \\
& \cong\left(L^{2}\left(\mathbb{R}^{+}\right) \otimes \operatorname{Span}\left\{\xi_{\varepsilon}^{(1)}\right\}\right) \oplus\left(L^{2}\left(\mathbb{R}^{+}\right) \otimes\left(\oplus_{k=2}^{\infty} \operatorname{Span}\left\{\xi_{\varepsilon}^{(k)}\right\}\right)\right) ;
\end{aligned}
$$

$\left(K^{(n)}=\right.$ corresponding ray of the star graph $\left.\Gamma\right)$ (hence $\left.L^{2}\left(K^{(n)}\right) \cong L^{2}\left(\mathbb{R}^{+}\right)\right), D_{\varepsilon}$ is the disk in $\mathbb{R}^{2}$ centered at the origin and with radius $\varepsilon$, and $\left\{\xi_{\varepsilon}^{(k)} \mid k \in \mathbb{N}\right\}$ is the o.n.b. of $L^{2}\left(D_{\varepsilon}\right)$ consisting of all Dirichlet Laplacian eigenfunctions.

We note that this decomposition is not left invariant by the flow of $H_{\varepsilon}$.

Consider the natural map:

$$
\Pi_{\varepsilon}: L^{2}\left(\Gamma_{\varepsilon}\right) \rightarrow L^{2}(\Gamma)
$$

which "crushes" the square integrable functions on the fat graph to square integrable functions on the star graph by first taking only the part of the function existing on the cylinders $K_{\varepsilon}^{(n)}$ 's and neglecting the part supported on the vertex region $\Omega_{\varepsilon}$, and then on each cylinder projecting the transversal part of the wave-function onto $\xi_{\varepsilon}^{(1)}$.

We want to investigate the limit of the "squeezed resolvent":

$$
\Pi_{\varepsilon}\left(H_{\varepsilon}-k^{2}\right)^{-1} \Pi_{\varepsilon}^{*} \stackrel{\varepsilon \rightarrow 0}{\longrightarrow} ?
$$

\subsection{Resonant case}

Resonant case: $\exists$ a zero energy resonance $\Phi_{\varepsilon}$ for $H_{\varepsilon}$ :

$$
\begin{aligned}
\Phi_{\varepsilon} & \in L_{\mathrm{loc}}^{2}\left(\Gamma_{\varepsilon}\right) \backslash L^{2}\left(\Gamma_{\varepsilon}\right) \\
H_{\varepsilon} \Phi_{\varepsilon} & =0 \quad \text { distributionally . }
\end{aligned}
$$

on the $n$-th cylinder, $K_{\varepsilon}^{(n)}$ it has the form

$$
\left(\left.\Phi_{\varepsilon}\right|_{K_{\varepsilon}^{(n)}}\right)\left(x_{n}, y_{n}, z_{n}\right)=\xi_{\varepsilon}^{(1)}\left(x_{n}, y_{n}\right)\left(a_{n}+b_{n} z_{n}\right) .
$$

Each self-adjoint Laplacian $\Delta_{A, B}$ on the star graph $\Gamma$ is identified by a vertex boundary condition on each $f \equiv\left(f^{(1)}, \ldots, f^{(N)}\right)$

$$
A\left(\begin{array}{c}
f^{(1)}(0) \\
\vdots \\
f^{(N)}(0)
\end{array}\right)+B\left(\begin{array}{c}
f^{(1) \prime}(0) \\
\vdots \\
f^{(N) \prime}(0)
\end{array}\right)=0
$$

for suitable $N \times N$ matrices $A$ and $B$ [Kostrykin-Schrader]. Each $\Delta_{A . B}$ admits a zero-energy resonance in $L_{\text {loc }}^{2}(\Gamma) \backslash L^{2}(\Gamma)$, that on each ray $K^{(n)}, n=1, \ldots, N$, behaves as $\alpha_{n}+\beta_{n} z_{n}$ for certain coefficient pairs $\left(\alpha_{n}, \beta_{n}\right)$ determined by $A$ and $B$.

There is an evident one-to-one correspondence between the set of parameters qualifying a resonance on the fat graph and the set of parameters qualifying a resonance on a star graph, an observation that we now intend to develop further.

Let $-\Delta_{A, B}$ be that Laplacian whose resonance's behavior is given by $\alpha_{n}=a_{n}, \beta_{n}=b_{n}$. 
It can be argued that if $\Pi_{\varepsilon} H_{\varepsilon} \Pi_{\varepsilon}-\left(-\Delta_{A, B}\right)$ has no further resonance, and if the resolvent convergence has suitable distributional properties then the limit exists and corresponds to $a$ Laplacian $-\Delta_{A, B}$ i.e. $\left.\Pi_{\varepsilon} H_{\varepsilon}-k^{2}\right) \Pi_{\varepsilon}^{*} \stackrel{\varepsilon \rightarrow 0}{\longrightarrow}\left(-\Delta_{A, B}-k^{2}\right)^{-1}$

"the limit is selected by the resonance of $H_{\varepsilon}$ ".

Note that this claim is well-posed, for the resonance function of $H_{\varepsilon}$ is scale invariant.

The claim is proved by the following argument. Let $H_{0} \equiv \Pi_{\varepsilon} H_{\varepsilon} \Pi_{\varepsilon}$ and let $V_{\varepsilon} W_{\varepsilon}^{*}=$ $H_{0}+\Delta_{A, B}$. One has:

$$
\begin{gathered}
\frac{1}{H_{0}+V_{\varepsilon} W_{\varepsilon}^{*}-z}-\frac{1}{H_{0}+z}=\frac{1}{H_{0}-z} V_{\varepsilon} C_{\varepsilon} W_{\varepsilon}^{*} \frac{1}{H_{0}-z} \\
C_{\varepsilon} \equiv \frac{1}{1-W_{\varepsilon}^{*} \frac{1}{H_{0}-z} V_{\varepsilon}}
\end{gathered}
$$

If $V_{\varepsilon}$ and $W_{\varepsilon}$ are $H_{0}$-compact and converge weakly to zero when $\varepsilon \rightarrow 0$ and if $H_{0}$ and $\Delta_{A, B}$ have the same zero-energy resonances, then $C_{\varepsilon}$ vanishes in the limit $\epsilon \rightarrow 0$.

\subsection{Non-resonant case}

Non-resonant case: no zero-resonance for $H_{\varepsilon}$.

Two subcases:

$$
\text { First case: } \quad \lambda_{\varepsilon}<\mu_{-}\left(\Omega_{\varepsilon}\right) \text {. }
$$

In this case, the energy threshold for the internal region is high (compared to $\lambda_{\varepsilon}$ ), which means that the domain $\Omega_{\varepsilon}$ has to be "very small" (in order for the spectrum of the internal region problem to have such a high bottom). Functions that belong to the continuous spectrum of $H_{\varepsilon}$ have a component in $\Omega_{\varepsilon}$ vanishing in the sup-norm as $\varepsilon \rightarrow 0$, in order for their $H^{2}$-norm to stay finite. Therefore, the functions in the domain of any limit operator on the graph must be zero at the vertex.

$\Rightarrow$ The limit is the Dirichlet Laplacian.

$$
\text { Second case: } \quad \lambda_{\varepsilon}>\mu_{+}\left(\Omega_{\varepsilon}\right) \text {. }
$$

Now the energy threshold for the internal region is low (compared to $\lambda_{\varepsilon}$ ) and the argument above does not apply. We expect that the projection $\Pi_{\varepsilon}$ "kills" in the limit the wave function (the fast transversal oscillations average to zero).

Therefore, in this case too, we expect that if the limit dynamics on the star graph exists they are Dirichlet-based.

But consider that strong convergence of the resolvents as bounded operators in the Hilbert space does not imply that the limit be the resolvent of a self-adjoint operator even if the convergence is strong and the resolvent identities are satisfied. The limit must be analytic away from the real axis and strong convergence need not preserve analyticity. We shall later give a simple example.

We expect that in the limit $\Pi_{\varepsilon}\left(H_{\varepsilon}-k^{2}\right)^{-1} \Pi_{\varepsilon}^{*}$ becomes analytic for $I m k^{2}>0$ and regular at $k^{2} \rightarrow 0$ as $\varepsilon \rightarrow 0$, because on the star graph, the Dirichlet Laplacian is the only self-adjoint Laplacian whose spectral measure is regular at zero [Kostrykin-Schrader].

A removal of singularity of the resolvent must therefore take place in the limit $\varepsilon \rightarrow 0$. This is typical of this second sub-case: in the first sub-case $\lambda_{\varepsilon}<\mu_{-}\left(\Omega_{\varepsilon}\right)$, instead, the resolvent of $H_{\varepsilon}$ is regular at $k^{2}=0$ uniformly in $\varepsilon>0$ and hence also in the limit. 
From "fat" graphs to metric graphs...

A way to monitor this removal of singularity for the limiting resolvent is to compare (the resolvent of) $H_{\varepsilon}$ with the second operator:

$$
H_{\varepsilon}+V_{\varepsilon} \quad V_{\varepsilon}:=C \varepsilon^{-2} \mathbf{1}_{\Omega_{\varepsilon}}
$$

We choose $C>0$, which is always possible, so that the "modified internal region problem" $-\Delta+V_{\varepsilon}$ on $\Omega_{\varepsilon}$, with a given boundary condition $\alpha$ at the bases of the cylinders and, as usual, Dirichlet boundary conditions on the remaining part of $\partial \Omega_{\varepsilon}$, has the lowest eigenvalue that precisely coincides with $\lambda_{\varepsilon}$ (the role of $V_{\varepsilon}$ is therefore merely to lift the bottom of the spectrum of the internal region problem up to the desired level $\lambda_{\varepsilon}$ ). For convenience, we denote by $\varphi_{\varepsilon}$ the corresponding lowest energy eigenfunction, that is, $\left(-\Delta+V_{\varepsilon}\right) \varphi_{\varepsilon}=\lambda_{\varepsilon} \varphi_{\varepsilon}$ in $\Omega_{\varepsilon}$ with Dirichlet boundary conditions on the whole $\partial \Omega_{\varepsilon}$.

The internal region problem, consisting now of the negative Laplacian plus the potential $V_{\varepsilon}$ with a given boundary condition $\alpha$, has the lowest eigenvalue that can be lifted up by means of a suitable choice of the constant $C$ so as to precisely match the value $\lambda_{\varepsilon}$. This does not alter, but for an overall phase factor, the solutions to the internal region problem and hence the matching conditions at the bases of the cylinders.

Notice:

$\sigma\left(H_{\varepsilon}+V_{\varepsilon}\right)=[0,+\infty)$

$H_{\varepsilon}+V_{\varepsilon}$ admits a zero energy resonance.

We thus have two operators on $L^{2}\left(\Gamma_{\varepsilon}\right)$, namely $H_{\varepsilon}$ and $H_{\varepsilon}+V_{\varepsilon}$, where the latter is a perturbation of the former and it is zero-resonant.

This is the input for a well-established scheme developed by Kato, Konno, and Kuroda that allows one to re-write:

$$
\left(H_{\varepsilon}+V_{\varepsilon}-\lambda\right)^{-1}-\left(H_{\varepsilon}-\lambda\right)^{-1},
$$

in a way that is well suited for taking the limit $\varepsilon \rightarrow 0$ and for taking advantage of the existence of a zero-energy resonance.

For instance:

$$
\begin{gathered}
\Pi_{\varepsilon}\left(\left(H_{\varepsilon}^{N}+V_{\varepsilon}-\lambda\right)^{-1}-\left(H_{\varepsilon}^{N}-\lambda\right)^{-1}\right) \Pi_{\varepsilon}^{*} \stackrel{\varepsilon \rightarrow 0}{\longrightarrow} \\
\stackrel{\varepsilon \rightarrow 0}{\longrightarrow}\left(-\Delta^{N}-\lambda\right)^{-1}+C(k) P_{N, k}
\end{gathered}
$$

where $P_{N, k}$ is the projection onto the vector $\mid G_{k}^{N}(., 0)>$.

Using the explicit form of the resolvents on a graph given e.g. in [1], one verifies that the last term is:

$$
\left(\left(-\Delta^{D}-\lambda\right)^{-1}-\left(-\Delta^{N}-\lambda\right)^{-1}\right)
$$

and therefore $\Rightarrow$ The limit is the resolvent of the Dirichlet Laplacian.

Notice: $V_{\varepsilon}$ is added in the internal region $\Omega_{\varepsilon}$ and this can be done only in the case of a fat graph $\Gamma_{\varepsilon}$. This procedure has no counterpart on the graph $\Gamma$ (because there is no internal region).

One may hope to obtain similar results by adding directly in the graph a potential $V_{\varepsilon}$ supported around the vertex of the graph and taking the limit when the support shrinks to the vertex.

Prototype: a "star graph" with one edge only. The corresponding fat graph has the shape of a safety match.

By choosing suitably the shape of the head of the safety match, one can produce in the limit any boundary condition at the vertex.

We can now try to change boundary conditions at the vertex of the graph by adding a 
potential shrinking around the origin

We introduce the Hamiltonian on $L^{2}\left(\mathbb{R}^{+}\right)$:

$$
\begin{gathered}
H_{\varepsilon}^{(\nu)}=-\Delta_{\nu}+V_{\varepsilon}, \quad \mathcal{D}\left(H_{\varepsilon}^{(\nu)}\right)=\mathcal{D}\left(-\Delta_{\nu}\right), \\
V_{\varepsilon}(x):=\frac{1}{\varepsilon^{1+\gamma}} V\left(\frac{x}{\varepsilon}\right),
\end{gathered}
$$

where

$\rightarrow V$ is real-valued, $V \in L^{1}\left(\mathbb{R}^{+}\right) \cap L^{\infty}\left(\mathbb{R}^{+}\right)$

$\rightarrow$ self-adjoint boundary condition at the origin

$$
f(0) \sin \nu=f^{\prime}(0) \cos \nu \quad \nu \in\left(-\frac{\pi}{2}, \frac{\pi}{2}\right]
$$

$\rightarrow \gamma<0$ weak scaling, $\gamma=0$ canonical, $\gamma>0$ strong

The problem is simple enough to allow an explicit solution.

For $k^{2} \in \mathbb{C}$ with im $\mathrm{k}_{\iota} 0$, we find that

$$
\left(-\Delta_{\nu}+V_{\varepsilon}-k^{2}\right)^{-1} \stackrel{\varepsilon \rightarrow 0}{\longrightarrow}\left(-\Delta_{\nu}-k^{2}\right)^{-1}+\Theta_{\nu, V, k}(\gamma)\left|\eta_{\nu, k}\right\rangle\left\langle\overline{\eta_{\nu, k}}\right|
$$

in the norm operator sense, where

$$
\Theta_{\nu, V, k}(\gamma)=\left\{\begin{array}{cl}
\eta_{\nu, k}(x):=(\tan \nu-k)^{-1} e^{i k x}, & x \in[0,+\infty), \\
0 & \text { if } \gamma<0 \text { (weak scaling) } \\
-\frac{\left(\int_{\mathbb{R}^{+}} V\right)}{1+(\tan \nu-\mathrm{i} k)^{-1}\left(\int_{\mathbb{R}^{+}} V\right)} & \text { if } \gamma=0 \text { (canonical scaling) } \\
-(\tan \nu-\mathrm{i} k) & \text { if } 0<\gamma<1 \text { (strong scaling). }
\end{array}\right.
$$

The convergence being in operator norm, the limit operator $R_{k}$ satisfies the resolvent identity.

In the weak scaling regime the boundary conditions are not changed.

In the canonical or strong scaling, in general $R_{k}$ is not the resolvent of a self-adjoint operator (in particular the limit does not produce new boundary conditions at $x=0$ ), because $R_{k}$ is not holomorphic in $k^{2}$ :

in fact $R_{k}=\left(-\Delta_{D}-k^{2}\right)^{-1}$ for all $k^{2} \in(-\infty, 0)$,

but $R_{k} \neq\left(-\Delta_{D}-k^{2}\right)^{-1}$ for some values $k^{2} \in \mathbb{C} \backslash(-\infty, 0)$

Exceptions:

$\rightarrow \int_{\mathbb{R}^{+}} V=0$ in the canonical scaling $(\gamma=0)$

$\rightarrow$ Dirichlet boundary conditions are preserved in any scaling

This phenomenon can be proved for a general metric graph as well.

Remarkably, the only exception is a "fake" star graph consisting of the real line $\mathbb{R}$ regarded as the union of the two rays $\mathbb{R}^{+}$and $\mathbb{R}^{-}$.

In this case one can add to the self-adjoint Laplacian on $\mathbb{R}$ a potential $\varepsilon^{-1} \mathbf{1}_{\{|x| \leqslant \varepsilon\}}$ at the "vertex" of the graph so to obtain in the limit $\varepsilon \rightarrow 0$ a so-called "point interaction" at the origin, namely a self-adjoint operator with certain boundary conditions at the origin [2].

\section{References}

[1] K. Kostrykin, S. Schrader. Kirchhoff's rule for quantum wires. J. Phys. A: Math. Gen., 1999, 32, P. 595-630.

[2] S. Albeverio, R. Høegh-Krohn. Perturbation of resonances in quantum mechanics. J. Math. Anal. Appl., 1984, 101(2), P. 491-513. 